\title{
Erratum
}

\section{Dimethyldioxirane (DMD)}

Vishnu Prabhakar Srivastava* Synlett 2008, 626.

This spotlight was inadvertently given No. 229. The correct labelling is Spotlight 231. 